Historic, Archive Document

Do not assume content reflects current scientific knowledge, policies, or practices. 



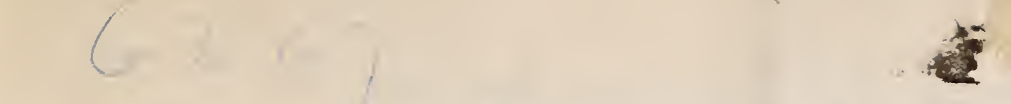

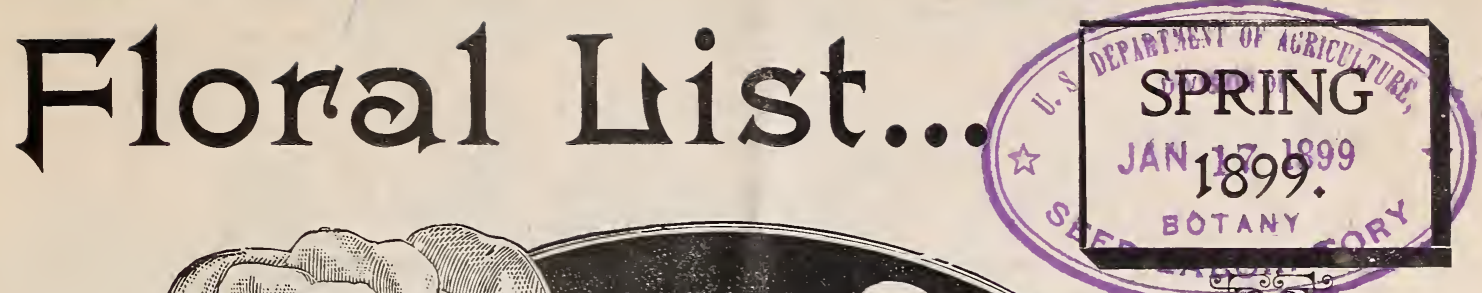
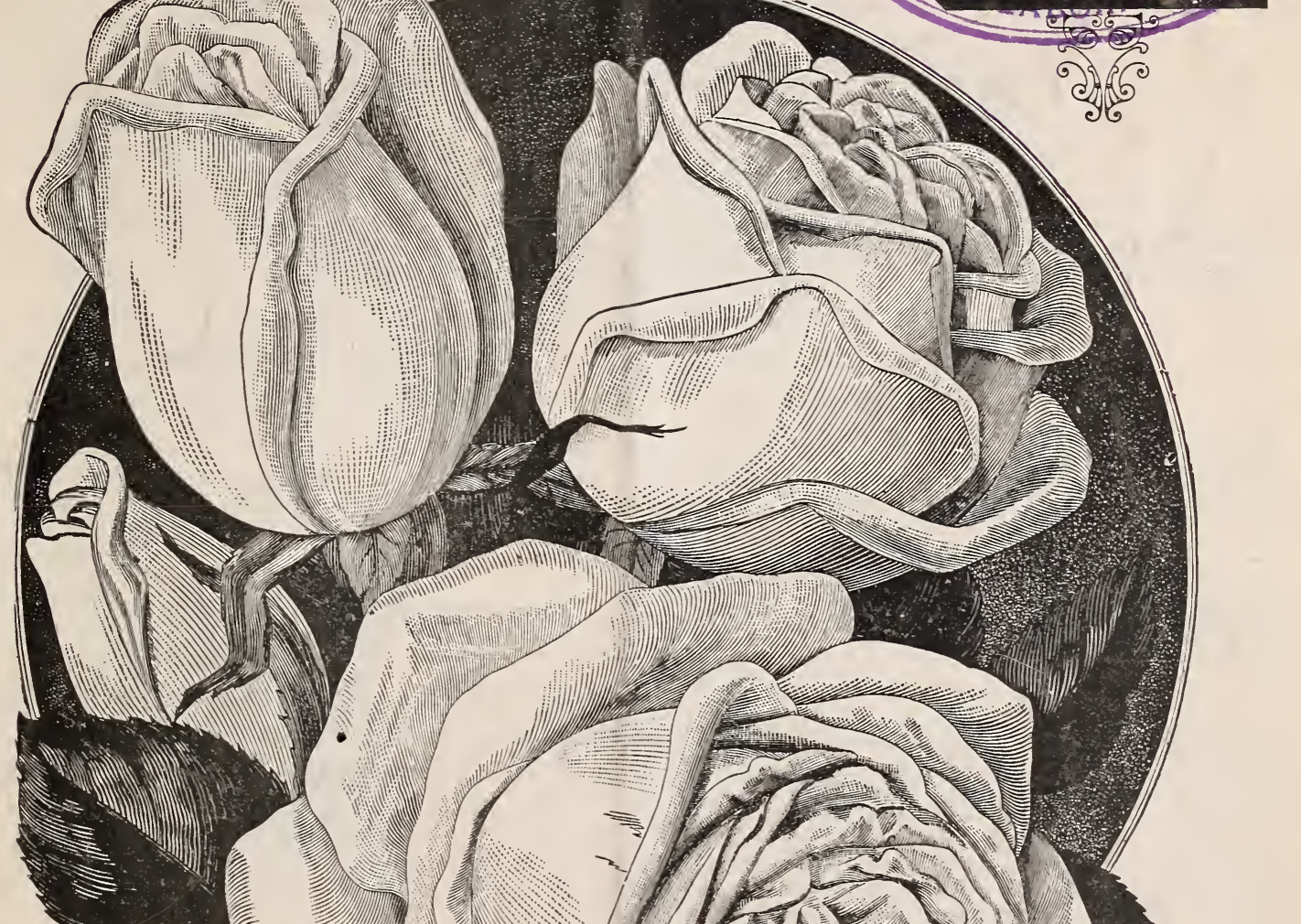


\section{Note Carefully, Our}

米

\section{Low Prices.}

\section{THE MONEY SAVED BY OUR NET PRICE LIST.}

As we have in the two preceding years, issued a net price list, thereby giving our patrons the benefit of a large amount of money which it costs to get out a fine illustrated catalogue; and as our plan has been so heartily approved by the many thousands of people who sent orders, we will do so again this season.

We thank all for their patronage and many kind words of praise for our plants.

$$
* *
$$

\section{THE QUALITY OF OUR STOCK STRICTLY THE BEST.}

Any who feared from our low prices that our stock might be inferior, were agreeably surprised to find that it was of the finest grade, and could not be excelled. This was shown us by the numerous orders received from the same people, and the many meritorious commendations we have from them. Our plants this season are finer than ever.

$$
\text { * } *
$$

\section{THE SIZE OF OUR STOCK IMMENSE.}

We have millions of plants on hand this season embracing only the best and most valuable varieties, such as we know will please all who receive them.

$$
\text { * } *
$$

\section{SEND IN YOUR ORDERS EARLY.}

As our low prices bring us thousands and thousands of orders, which are filled in the order they are received.

REMEMBER THE NEW LOW EXPRESS RATE. You will bear in mind that the rate on plants is 20 per cent. less than the regular merchandise rate.

\section{OUR ROSES ARE ALL ON THEIR OWN ROOTS.}

Yours sincerely,

\section{Che Dational Plant Company,}




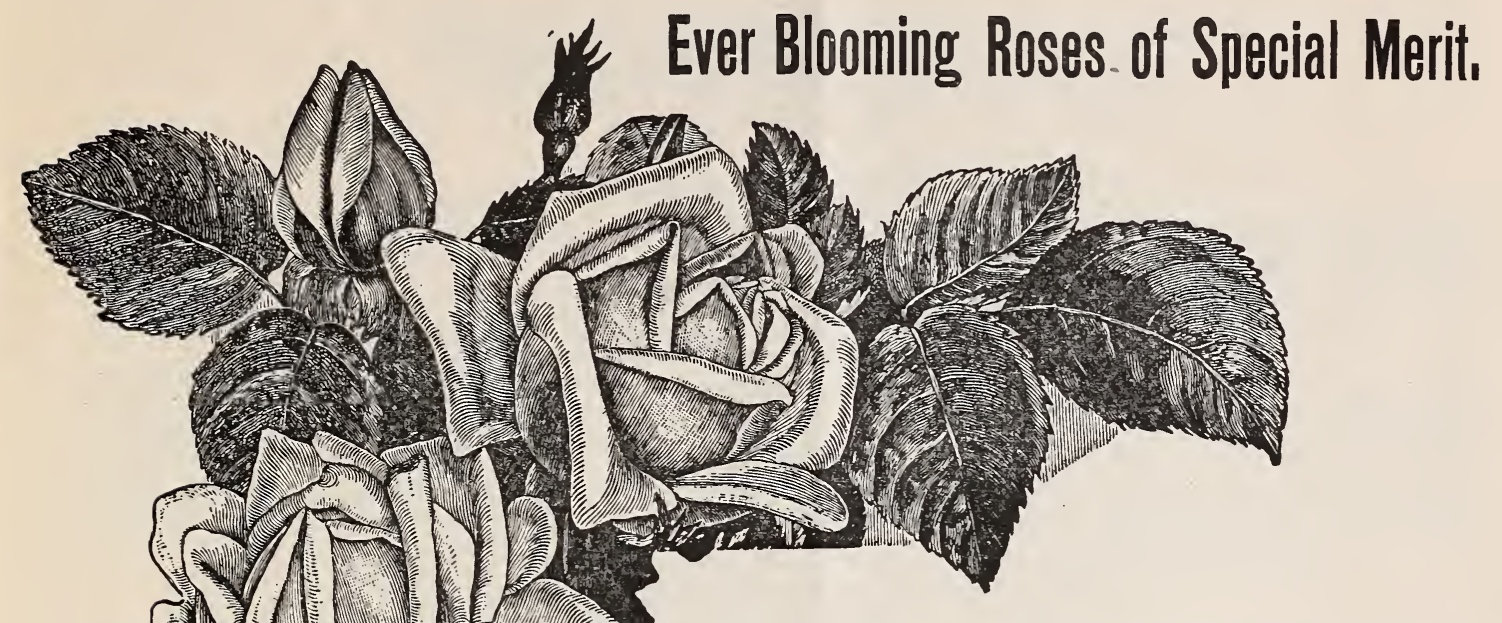

Pink Rambler-Grand new hardy Pillar Rose. A most vigorous grower, of beautiful foliage. Flowers in great clusters. Color a most dainty shiny rose. A very free bloomer, and equal to Crimson Rambler. 7 cents each, large size 15 cents.

White Rambler-In habit of growth, foliage and manner of bloom, also hardiness, it is identical with the Crimson Rambler, of which it is said to be a blood relation. The flowers are pure snow white, and sweetly scented. 7 cents each, large size 15 cents.

Yellow Rambler- The hardiest yellow climbing rose out. Flowers in immense clusters after the manner of Crimson Rambler. Handsome foliage, and flowers a beautiful decided jellow. Very free in bloom, and hard to equal. 7 cents each, large size 15 cents.

Golden Rambler-_" Allister Stella Gray," a new climbing rose, in habit very similar to the other ramblers. Flowers in large clusters, pretty straw-color and orange yellow. 7 cents each, large size 15 cents.

President Carnot.

Climbing White Pet-A most beautiful, white hardy climber. Pure white flowers, very free in bloom, and flowers in great bunches. One of the grandest acquisitions. 7 cents each, large size 15 cents.

Climbing Malmaison-A rapid hardy climber, possessing all the qualities of the grand old Malmaison, the fine bedding rose. Flowers in the hottest weather, perfect, rich, flesh-colored, and in great abundance. A noble rose, 7 cents each, large size 15 cents.

Climbing Wootton-A free climber, with the qualities of the old stand-by Wootton. This superb rose has rich, glossy, large foliage, and is a very free bloomer. Flowers velvety red, full and double, and very sweet scented. Quite hardy. One of the best. 7 cents each, large size 15 cents.

President Carnot-A charming, new, hardy French rose. A strong, clean, healthy grower, with handsome bronzy-green foliage, large, fine shaped buds on long, erect stems. Color, delicate rosy-flesh, a trifle deeper at center. A very profuse bloomer. 7 cents each, large size 15 cents.

Memorial Rose-“"Wichuriana"-A trailing species of rapid growth, creeping close to the earth. The great cemetery rose. For rockery or shrubbery it has no equal. Flowers pure white and fragrant, in great profusion. Very hardy. 7 cents each, large size 15 cents.

Yellow Soupert_"Mosella," a valuable new Polyantha. Flowers as large as Clothilde Soupert, very double, and well shaped. Color, beautiful light yellow. Plant dwarf, bushy and very free flowering. 7 cents each, large size 15 cents.

English Sweetbrier Rose, Lord Penzance-A fine favorite for shrubberies and general planting. Flowers pretty fawn color. The fragrance of the flowers, leaves and young branches frequently perfume a neighborhood, and is delightful. Hardy. 7 cents each. Large size 15 cents.

English Sweetbrier Rose, Lady Penzance-Possesses all the fragrance of flowers, foliage and branches of the above. Flowers a beautiful coppery yellow. A grand rose. Hardy. 7 cents each, large size 15 cents.

Clio-New hardy Rose of great beauty. Handsome flowers of large globular form in great abundance. Color, delicate flesh. In all, the finest white Hybrid up to date. 7 cents each, large size 15 cents.

Mrs. Robert Garrett-The new Hybrid Tea. One of the most beautiful pink roses ever introduced. 15 cents each, large size 30 cents. 


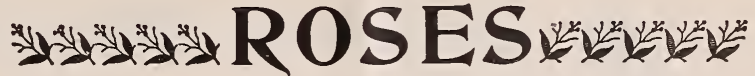

STRONG YOUNG PLANTS.

Your selection from our entire list, labeled, by mail................... cents each, 20 for $\$ 1.00$ Our selection from our entire list, all different, labeled, by mail.......................25 for $\$ 1.00$ Our selection from our entire list, all different, not labeled, by mail....................30 for $\$ 1.00$

\section{LARGE SIZE PLANTS.}

Your selection from our entire list, labeled, by mail...................10 cents each, 10 for $\$ 1.00$ Our selection from our entire list, labeled, by mail............................12 for $\$ 1.00$ Your selection from our entire list, labeled, by express.......................... 12 for $\$ 1.00$ Our selection from our entire list, labeled, by express............................15 for $\$ 1.00$ Our selection from our entire list, not labeled, by express........................20 for $\$ 1.00$ When ordered by express, larger plants will be sent than can be forwarded through the mails. All plants, either by mail or express, strictly first-class.

\section{EVER=BLOOMING ROSES.}

Belle Siebrecht-Imperial pink.

Mrs. Pierpont Morgan-Bright cerise.

Mrs. W. C. Whitney-Deep pink.

Pink Soupert-Pink.

Mme. Elie Lamber-Creamy white.

Beaute Inconstant-Changeable orange-scarlet.

Golden Gate-Creamy white and gold.

Pierre Guillot-Rich crimson.

Striped Malmaison-Creamy white and rose, striped.

Mme. Pernet Duchere-Canary yellow and copper.

Viscountess Folkstone-Whitish flesh.

Madam Hoste-Beautiful cream.

Malmaison-Rich flesh.

Media-Golden yellow.

Etoil de Lyon-Golden yellow.

Mme. Cecil Berthod-Deep yellow.

Isabella Sprunt-Canary yellow.

Perle des Jardins-Faultless rich yellow.

Comtesse Frigneuse-Deep gold yellow.

Christine de Noue-Brilliant red.

Sunset-Golden yellow and copper.

Madam Cusin-Deep purplish rose.

Mme. Watteville-Yellow and blush.

The Queen-Snow white.

Duchess de Brabant-Rose and amber.

J. B. Varrone-Scarlet crimson.

Sappho-Yellow and buff.

Marie Guillot-Pure white.

Miss Wenn-Coral pink.

Madam Welche-Amber yellow.

Mme. Franciska Kruger-Deep coppery yellow.

Niphetos-Elegant white.

Mrs. DeGraw-Deep rosy pink.

Snowflake-Pure white.

Mme. Jos. Schwartz-White and pink.

Maman Cochet-Pink and rose.

Kaiserin Augusta Victoria-Creamy white.

Mme. Caroline Testout-Brilliant pink.

Bridesmaid-Rich pink.

The Bride-Spotless white.

Cornelia Cook-Creamy white.

Catherine Mermet-Pink.
Souvenir de Wootton-Velvety. red.

Pink La France-Pink.

White La France-White.

Red La France-Dark deep pink.

Striped La France-Striped pink and white.

Hermosa-Soft pink.

Yellow Hermosa-Rich yellow.

White Hermosa-Pure white.

Clothilde Soupert-From soft pink to pure white.

Papa Gontier-Deep crimson.

Champion of the World-Rich gloss pink.

Crown Princess Victoria-Waxy snow-white.

Marie Van Houtte-Faultless straw-yellow.

Senator. McNaughton - The white "Perle."

Meteor-Rich velvety red.

Mme. Bravy-Creamy white.

Madam Caroline Kuster-Pale yellow.

Etoile de Angers-Chamois yellow.

Aggrippina-Rich crimson

Camoens-Rich pink.

Marquis de Viviens-Carmine and rose.

Andre Schwartz-Glowing crimson.

Abbe Girardin-Satiny rose.

Riza du Park-Deep carmine.

La Pactole-Cream, center yellow.

Pink Rover-Tender rose.

Louisa de la Rive-Soft rose-blush.

Lucullus-Velvety crimson.

Lady Warrender-Pure white.

Louis Richards-Rose salmon.

Dr. Dusillet-Salmon yellow.

Marie Ducher-Delicate pink.

Melville-Bright pink.

Madam Bosanquet-Flesh color.

Appolline-Bright pink.

Purple China-Crimson.

Sombreuil-White-tinged rose.

Edouard Littaye-Rosy crimson.

Elise Hayman-Light yellow.

Princess Sagan-Bright scarlet.

Duchess of Edinburgh-Intense crimson.

Mme. Veuve Menier-Light rose.

Grand Duchess Hilda-Nankeen yellow. 


\title{
EVER=BLOOMING ROSES-Continued.
}

\section{Prices Same as On Previous Page.}

President-Soft crimson.

Rubens-Pale yellow.

Progress-Rosy carmine.

Dr. Passott-Velvety carmine.

Triumph de Pernet Pere-Bright, red.

Rainbow-striped.

Amanda Casado-Rose and chamois.

Henry Brichard-White and rose.

Gustave Nadaud-Vermillion.

Marechal Robert-White-tinged blush.

Beauty of Stapleford-Bright pink.

Antoine Verdier-Silvery rose.

Souv. de Un Ami-Rosy flesh.

Eva Starhemburg-Creamy Yellow.

Buchess Marie Salviatia-Orange.

Eliza Fugier-Creamy white.
Georges Farber-Velvety purple.

Safrano-Apricot yellow.

Mme. Margottin-Citron yellow.

Mme. Lombard-Rosy salmon.

Mme. Phillippi Kuntz-Cherry red.

Madam Olga-Rich cream-white.

May Rivers-White, center yellow.

Maurice Rouvier-Light pink and rose.

Miss Marston-Creamy white.

Adam-Salmon rose.

Anna Ollivier-Buff-shaded rose.

Bon Silene-Deep Salmon Rose.

Capitaine le Fort-Rosy purple.

Queen's Scarlet-Velvety scarlet.

Bona Weillshott-Rosy vermillion.

Lady H. Grosvenor-Rich flesh.

\section{CLIMBING EVER-BLOOMERS.}

Gloire de Dijon-Rich rose and yellow.

Climbing La France-Richest pink.

Reine Marie Henriette-Pure cherry red.

Cloth of Gold-Golden yellow.

Lamarque-Pure white.

William Allen Richardson-Orange yellow.
Climbing Devoniensis-Cream-tinged blush.

Climbing Perle des Jardins-Richest yellow.

Climbing Niphetos-Pure white.

Marechal Niel-Golden yellow.

Solfaterre-Sulphur yellow.

\section{HARDY CLIMBING ROSES.}

Baltimore Belle-Beautiful white.

Mme. Gustave Gossard-Light pink.

Prairie Queen-Clear bright pink.

Tennessee Belle-Clear rose.
Seven Sisters-Varies white to red.

Mary Washington-Pure white.

Crimson Rambler-Bright crimson.

Empress of China-Fine pink.

\section{HARDY ROSES.}

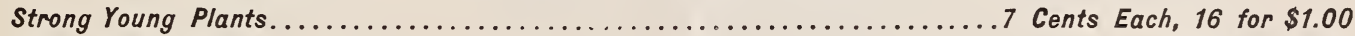

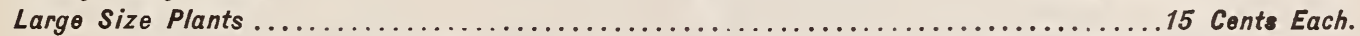

When ordered by express, larger plants will be sent than can be forwarded through the mails. All plants, either by mail or express, strictly first-class.

La France de '89-Bright scarlet.

Victor Verdier-Cherry rose.

Glorie Lyonnaise-Chamois yellow.

Madam Charles Wood-Fiery scarlet.

General Washington-Soft scarlet.

General Jacqueminot-Bright crimson

Boule de Neige-Pure white.

Fisher Holmes-Rich carmine.

Margaret Dickson-Fine white.

Paul Neyron-Deep rose.

Gloire de Bruxelles-Bright crimson.

Gloire de Margottin-Dazzling red.

Gustave Piganeau-Brilliant crimson.

Mme. Gabriel Luizet-Coral rose.

Anna des Diesbach-Carmine.

Alfred Colomb-Cherry red.

Charles Lefebvre-Dark crimson.

Glory of Paris-Brilliant crimson.

Jules Margottin-Cherry red.

John Hopper-Rich rose.

Louis Van Houtte-Crimson maroon.

La Reine-Bright rose.

Giant of Battles-Brilliant crimson.
Duke of Edinburgh-Dark maroon.

Mme. Alfred de Rougemont-Pure white.

Marie Bauman--Rich red.

Magna Charta-Rosy red.

Mme. Plantier-Purest white.

Pierre Notting-Deep crimson.

Heinrich Schultheis-Delicate rose.

Earl of Dufferin-Crimson.

Coquette des Alps-Pure white.

Countess of Oxford-Carmine red.

Duke of Connaught-Blackish red.

Black Prince-Blackish red.

Elizabeth Vegneron-Shell pink.

Eugene Verdier-Silvery pink.

Ferdinand de Lessepps-Bright crimson.

Eugene Fuerst-Crimson scarlet.

Comte de Paris-Bright crimson.

Prince Camille de Rohan-Dark crimson.

Ulrich Brunner-Cherry red.

Mme. Thibaut-Bright cherry red.

Martin Cahuzac-Rose carmine.

Marcbioness of Lorne-Soft carmine.

Coquette des Blanches-Pure white. 


\section{BEST GERANIUMS.}

\section{STRONG YOUNG PLANTS.}

Your selection from our entire list, labeled, by mail....................5 cents oach, 20 for $\$ 1.00$ Our selection from our entire list, all different, labeled, by mail......................25 for $\$ 1.00$ Your selection from our entire list, labeled, by express. . . . . . . . . . . . . . . . . . . . 25 for $\$ 1.00$ Our selection from our entire list, all different, labeled, by express................... 30 for $\$ 1.00$ When ordered by express, larger plants will be sent than can be forwarded through the mails. All plants, either by mail or express, strictly first-class.

\section{SINGLE GERANIUMS.}

Columbia-Scarlet and white.

L. Swartland-Salmon.

W. T. Gunn-Red and white.

M. Porier-Carmine violet.

Souv. de Mirande-White and rose.

Copernic-Rosy carmine.

Mrs. E. G. Hill-Salmon.

Rev. Atkinson-Bright scarlet.

Rev. H. Harries-Rosy red.

Comt. de Pot-Salmon flesh.

Ensign-Bright scarlet.

Marguerite de Layres-Pure white.

Leon Perrault-Brilliant scarlet.

Francois Arago- Silvery salmon.

Mme. Guilbert-Pure rose.

Lillian Smith-Dazzling scarlet.

S. G. Cobb-Pink-tinged white.

Alcibiade-Red-salmon.

La Vestale-Pure white.

Louis Ulbach-Dazzling scarlet.

Lady R. Churchill-Deep salmon.
Ben Schroder-Handsome pink.

Gen. Dodds-Soft vermillion.

Gen. Sheridan-Bright scarlet.

Earnest Bergman-Velvety scarlet.

Queen Olga-Deep pink.

Mrs. J. M. Garr-Rich white.

Queen of the West-Orange-scarlet.

Sam Sloan - Crimson.

Blanche Moulas - Salmon and white.

Mrs. Lindsay-Pink-violet.

Mary H. Foote-Salmon and white.

Baron Puynode-Orange-vermillion.

Leviathan-Crimson scarlet.

James Kelway-Orimson scarlet.

Mrs. Hamilton-Soft pink.

Aurelia Scholl-Violet rose.

Trophee-Rich lilac.

Frau Louise Voith-Clear pink.

La Lorraine Tender flesh.

Madonna-Pale pink.

Wood Nymph-Clear pink.

\section{DOUBLE GERANIUMS.}

Dr. Blanche-Orange scarlet.

L. Contable-Bright rose.

White Swan-Pure white.

Hetheranthe-Bright red.

Emile de Girardin-Deep pink,

Emerson-Nankeen salmon.

S. A. Nutt-Dark crimson.

Beauty Poitevine-Brilliant salmon.

Harriet Thorpe-Delicate blush.

Mme. Dieulafoy-Tender rose.

Chaplain-Vermillion red.

Gripper Banks-Dark crimson.

Re Umberto-Orange scarlet.

Le Pilote-Rich crimson.

James Vick-Deep flesh.

Gold Dust-Bright scarlet.

C. A. Knorr-Lilac red.

Dr. Thouvenet-Rosy scarlet.

De Lacepede-Rose salmon.

Mathias Sandorf-Reddish salmon.

Black Knight-Crimson maroon.

Alfred Tennyson-Scarlet.

Monsieur G. Lowaige-Bright scarlet.

Barbizet-Bright rose.

Madam Dupont-Rich scarlet.

A. Dupree-Velvety crimson.

Peter Henderson-Orange.

Bossingalt-Orange vermillion.

M. Press-Rosy salmon.

Mme. La Baume-Rich rose.

Bonnat-Brilliant carmine.

Adrienne Corret-Reddish orange.

M. Louis Fages-Clear orange.

Montesquin-Light pink.

Louis Fihol-Reddish salmon.

\section{SWEET-SCENTED GERANIUMS.}

Balm.

Shrubland Pet.

Nutmeg.

Lemon-Scented.

Mrs. Taylor.

Oak Leaf.
Peppermint.

Pennyroyal.
Dr. Livingston.

Rose.

\section{GOLDEN BRONZE GERANIUMS.}

Exquisite-Salmon and white.

Fanny-Peach.

Prince Bismarck-Salmon.

Zulu-

\section{SILVER-LEAVED GERANIUMS。}

Mountain of Snow-Bright scarlet.

Happy Thought-Bright scarlet.

Mme. Salleroi-Dark red. 
IVY GERANIUMS.

Peter Crozy-Brilliant scarlet. Charles Turner-Light scarlet.
Joan of Arc-Pure white. Flourens-Salmon rose.

Galilee-Deep pink.
M. Claeys-Wine color. Innocence-Pure white. Eclipse-Bright rose.

\section{CHRYSANTHEMUMS.}

\section{STRONG YOUNG PLANTS.}

Your selection from our entire list, labeled, by mail......................5 cents each, 20 for $\$ 1.00$

Our selection from our entire list, labeled, by mail. ...........................25 for $\$ 1.00$

Your selection from our entire list, labeled, by express.......................... 25 for $\$ 1.00$

Our selection from our entire list, labeled by express. . . . . . . . . . . . . . . . . . . . 30 for $\$ 1.00$

When ordered by express, larger plants will be sent than can be forwarded through the mails. All plants, either by mail or express, strictly first-class.

G. W. Childs-Deep velvety crimson.

Viviand Morel-Light creamy rose.

A. J. Drexel-Dark red.

Ivory-Pure white.

Wm. Simpson-Fine early pink.

White Swan-Beautiful white.

John Shrimpton-Bright crimson.

Octoroon-Handsome red.

Golden Hair-Fine yellow.

Fisher's Torch-Bright red.

Pink Ivory-Fine pink.

Glory of Pacific-Grand early pink.

Montgolfier-Maroon-tipped yellow.

Arbre de Noel-Deep orange shaded red.

Niveus-Snow white.

Georgianna Bramhall-Soft yellow.

Golden Bedder-Clear golden yellow.

White Bedder-Pure white.

Major Bonnaffon-Golden yellow.

Cravaction-Pink, yellow center.

Canary Bird-Light canary yellow.

Bouquet-White, tinged yellow.

Baron Veillard-Large brilliant yellow.

Mrs. Craig Lippincott-Golden yellow.
Pluto-Bright red, reverse bright golden.

W. H. Lincoln-Large bright yellow.

Gettysburg-Deep crimson.

Mrs. Perrin-Fine satin pink.

Titian-Bright pink.

Col. W. B. Smith-Golden bronze

W. G. Newitt-Fine white.

Mrs. S. Murdoch-Deep Pink.

Achilles-Soft pink.

Fred Waltz-New pink.

Mrs. E. G. Hill-Pink.

Mrs. Aristee-Deep canary yellow.

Chas. Pratt-Fine pink.

Pitcher \& Manda-Yellow, tinged white.

Mme. B. Jung-Large crimson red.

M. Lamaille-Large, wine color.

Marion Henderson-Fine early yellow.

J. E. Lager-Bright yellow.

Egeta-Fine Brilliant yellow.

W. B. Dinsmore-New yellow.

The Queen-Purest white.

Yellow Queen-Nice yellow.

Miss Florence Pullman-Fine new white.

Mutual Friend-Snowy white.

\section{OSTRICH PLUME CHRYSANTHEMUMS.}

Eiderdown-Large snow white.

W. A. Manda-Fine yellow.
Enfant des Mondes-Purest white.

Mrs. Higginbotham-Rich bright pink.

\section{BEST ABUTILONS.}

\section{PRICES SAME AS FOR CHRYSANTHEMUMS ABOVE.}

Souvenir de Bonne-Orange red. Arthur Belsham-Crimson. Boule de Neige-Pure white. Splendens-Deep red.
Sensation-Orange red.

Thompsonii Plena-Rich orange.

Eclipse-Bright yellow.

Royal Scarlet-Dark red.

Prince of Me'bourne-Orange striped.
Robert George-Bright orange.

Purpuria-Light violet.

Vesuvius-Velvety crimson.

Golden Bells-Golden yellow。

\section{New Low Express Rate.}




\section{CHOICE CARNATIONS.}

\section{STRONG YOUNG PLANTS.}

Your selection from our entire list, labeled, by Mail. 5 cents each, 20 for $\$ 1.00$ Our selection from our entire list, labeled, by mail. ..............................25 for $\$ 100$ Your selection from our entire list, labeled, by express.

When ordered by express, larger plants will be sent than can be forwarded through the mails. All plants, either by mail or express, strictly first-class.

Flora Hill-Pure white.

Mrs. Geo. Bradt-Striped.

Mrs. Chas. Duhme-Light pink.

Uncle John-Pure white.

E. A. Wood-Light pink.

Sweetbrier-Clear pink.

Wm. Scott-Deep pink.

Fred Dorner-Rich scarlet.

Grace Darling-Peach blossom.

Albertini-Flesh pink.

Tidal Wave-Rosy pink.

Jacqueminot-Rich crimson.

Golden Gate-Golden yellow.
Orange Blossom-Rose and white.

Mrs. Hitt-Deep pink.

Daybreak-Pure flesh.

Creole-Darkest red.

White Dove-Pure white.

Grace Wilder-Carmine pink

Hinze's White-White.

Silver Spray-Pure white.

President Garfield-Scarlet.

Portia-Bright scarlet.

Lizzie McGowan-Pure white.

Blanche-Pure white.

Ben Hur-Bright pink.

\section{FUCHSIAS.}

\section{STRONG YOUNG PLANTS.}

Your seleetion from our entire list, labeled, by mail...................5 cents each, 20 for $\$ 1.00$

Our selection from our entire list, labeled, by mail............................25 for $\$ 1.00$

Your selection from our entire list, labeled, by express........................... 25 for $\$ 1.00$

Our selection from our entire list, labeled, by express........................... 30 for $\$ 1.00$

When ordered by express, larger plants will be sent than can be forwarded through the mails.

All plants, either by mail or express, strictly first-class.

\section{DOUBLE FUCHSIAS.}

Phenomenal-Coral red.

Jupiter-Light magenta.

Rosains Patri-White.

Admiral Courbet-Violet.

Avalanche-Violet purple.

Champion of the World-Dark purple.

Duke of Albany-Lilac.

Mme. Van der Strauss-White.

Monstrosa Superba-White.

Molesworth-Pure white.

Sapaly Freres-Carmine red.

Mrs. S. M. Thomas-Dark purple.

Purple Prince-Deep blue.

\section{SINGLE FUCHSIAS.}

Speciosa-Brilliant carmine.

Arabella-Rose.

Pres. McMahon-Scarlet.

England's Glory-Carmine pink.

May Queen-Violet rose.

Annie Earle-Carmine.

Lovely-Crimson.

Enfant Prodigal-Purple.

De Mirble-Violet rose.

Black Prince-Pale carmine.

\section{Chinese Hibiscus, 8 cents each.}

Auriantica-Orange.

Carminatus Perfectus-Carmine.

Collerii-Yellow.

Grandiflora-Scarlet.

Miniatus Semi-Plena-Vermillion.

Rosa Siensis-Red.

Sub Violaceous-Carmine.

Versicolor-Striped.

Zebrinus-Scarlet and white.

Cooperi-Red.
Hardy Pinks, 5 cents each, 50c, per doz.

Alma Fimbriata-Pure white.

Anne Boleyn-Maroon.

Brunetta-Dark red.

Earle of Carlisle-Striped.

Juliette-Variegated red.

Gertrude-White.

Laure Wilmer-Red and white.

Stansalarius-Pink.

Her Majesty-Pure White.

Heliotropes, 5 cents each, 50 cents per dozen.

Black Knight-Dark Purple.

Mme. Carriere-Bright blue.

Julietta-Dark purple.

White Lady-Pure White.
Mme. Bruant-Rich purple.

Albert Delaux-Lavender.

Dark Bouquet-Dark blue. 


\section{FINEST BEDDING PLANTS.}

\section{NEW AND CHOICE LARGE-FLOWERING CANNAS.}

Nothing can be more effective for grouping on lawns or for large beds in parks, than these ever-blooming Cannas. They commence to flower in a very short time after vlanting, and are a mass of gorgeous colors until stopped by frost in Fall. 10 cents each, $\$ 1.00$ per dozen

Chas. Henderson - Strong green foliage, flowers scarlet-crimson.

Flamingo-Rich green foliage, flowers fiery crimson.

Paul Marquant-Foliage green, flowers large bright salmon with crimson tint.

Madam Crozy-Foliage green, flowers large brilliant scarlet bordered with golden jellow.

J. D. Cabos-Dark maroon foliage with bronze luster, flowers bright orange or apricot.

Captain DeSuzzoni-Foliage green and massive, flowers rich yellow spotted red.

Secretary Stewart-Foliage green, flowers bright garnet.
Queen Charlotte - Foliage heavy bluish-green, flowers rich crimson banded with canary yellow.

Florence Vaughan-Foliage massive green, flowers golden yellow, spotted with bright red.

Alphonse Bouvier-Rich green foliage, flowers rich dazzling crimson.

J. C. Vaughan-Foliage rich bronze-purple, flowers deep vermillion orange.

Egandale-Foliage dark bronze, flowers bright cherry-red.

Eldorado-Fine green foliage, flowers pure golden yellow, finely speckled with light red.

\section{OUR BEAUTIFUL NEW COLEUS.}

Coleus will give more pleasure at less cost than any other plant. Their varied tints of crimson, gold, bronze and green, and richly blotched, veined or margined, produce a brilliancy of coloring unequaled. Our list includes all the best standard kinds, such as Louise Beck, rich maroon; Gem City, golden-yellow spotted carmine; Golden Verschaffelti, bright golden yellow ; Firebrand, fiery red; Yeddo, jellow blotched with green; Clouded Gem, crimson and cream with green edge; Progress, mottled red, green and bronze, Mme. Mageau, wine color edged with gold and green; Octoroon, green and gold with bright red shading; Petit Robert, maroon cut and margined yellow and green; Brightness, vermillion blotched with chocolate and green; Retta Kirkpatrick, bright green, yellow center; Setting Sun, crimson with golden edge; Verschaffelti, rich velvety crimson, the finest of all dark varieties; Rag Carpet, carmine edged chocolate red ; Golden Bedder, bright golden yellow. Africa, Black. 30 cents per dozen, $\$ 2.00$ per 100 .

We call special attention to HETHERANTHE, the best of all bedding Geraniums. Foliage strong and striking, and of most perfect habit. Flower is a bright vermillion, of most pleasing shade, trusses exceptionally large and fine. Excellent for beds or pot. Try a bed of these. They will delight you.

Also Emile DeGirardin, the fine pink Geranium; L. Contable, beautiful rose color; White Swan, spotless white, and Mountain of Snow and Madem Salleroi, the two leading silver leafed Geraniums. 40 cents per dozen, $\$ 3.00$ per 100 .

\section{ALTERNANTHERA.}

Alternantheras are the best plants used for "carpet-bedding," massing or ribbon line planting or general bedding. Their unique form and tints, and the uniform manner in which they always grow, make them unequaled for fine bedding work, and a garden is incomplete without their finishing touches. We grow them in immense quantities, and have both the red and yellow. 30 cents per dozen, $\$ 2.00$ per 100 .

\section{PALMS.}

Kentia Fosteriana-The Kentias are among the best of the palms for general cultivation, being very free from disease of any kind. This variety is graceful in habit, with bright green foliage, and as a decorative plant for the window, dinner table or conservatory, it scarcely has an equal. Price 25 cents each.

Kentia Balmoreana-This is sometimes called the "Curly Palm." and is recognized as among the best for all purposes, being capable of standing more ill-usage, perhaps, than any other. The leaves have a tendency to curl as the plant grows older, hence its popular title of "Curly Palm." Price 25 cents each.

Filifera Palm-(Washington Filifera). It has elegant dark green fan-shaped leaves, from which hang thread-like filaments. Price 15 cents each. The plant is a compact grower, well adapted to pot culture.

Latania Barbonica-The well-known favorite Fan Palm, beautiful in all the stages of growth. No plant is more easily grown, and none more tenacious of life, enduring alike the dust, the cold and heat from open windows and gas-heated air of our dwellings. Price 15 cents each.

Phcenix Reclinata-This is one of the most exquisitely graceful among palms. Its wide-spreading gracefully-arching, fern-like leaves are elegant and effective for apartment decoration. It will stand much neglect without injury ; fine for vases. Price 15 cents each. 


\section{BEGONIAS, the Great Foliage Plants. \\ STRONG YOUNG PLANTS.}

Your selection from our list, labeled, by mail...........................8 cents each, 15 for $\$ 1.00$

Our seiection from our list, labeled, by mail. 20 for $\$ 1.00$

When ordered by express, larger plants will be sent than can be forwarded through the mails. All plants, either by mail or express, strictly first-class.

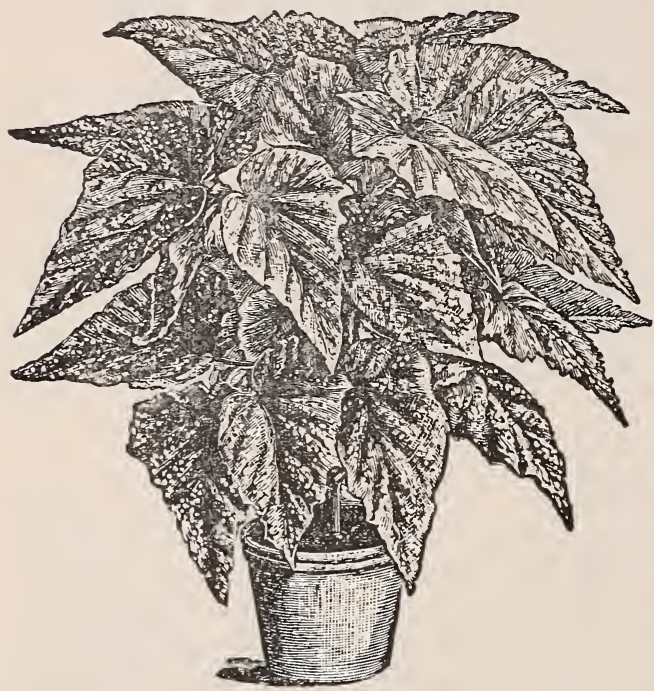

FLOWERING VARIETIES.

Vernon-New bedding Begonia. Foliage fine green, spotted and margined with bronzy-red. Very free bloomer. Flowers brilliant deep carmine.

Eufurt-White. Rich green foliage. Rapid grower. Flowers freely in clusters of pure white. One of the best bedding varieties.

Eufurt-Pink-Splended bedder. Foliage same as white Eufurt. Flowers very freely in clusters, color handsome light pink.

Dewdrop-Fine bedding Begonia. Rich green foliage. Profuse bloomer. Flowers a splendid white.

Bertha Chateaurocher - Shining green leaves. Will stand much sunlight. The flowers of bright currant red are in abundance

M. DeLessepps-Large purple bronze leaves beautifully spotted with silver. Strong and free grower. Color pure white.

President Carnot-Foliage very large, upper side deep green, under side purplish red. Flowers beautiful coral-red in clusters.

Argentea Guttatta-Large oblong purple bronze leaves with bright silvery blotches and markings. Beautiful white flowers. One cf the finest of all Begonias.

Robusta-Leaves bronze green with metallic luster. Free flowers, part white, part pink with pretty pink stripe.

Speculata-Foliage of Rex type, large and formed like a grape leaf, green and chocolate in color. Magnificent pink flowers lifted high above the foliage.

Rubra - The best grower of all. Waxen green heavy foliage, literally covered at all times with handsome coral-red flowers. A most wonderful plant. You should have this.

Hybrida Multiflora - Small bright shining foliage, with graceful branches and abundant flowers of beautiful shell-pink. Free bloomer.

Feastii-Creeping Begonia with perfectly circular leaves, red beneath and olive-green above. Flowers of most exquisite pink raised on long stems. Rapid grower of easy culture.

Sandersonii-One of the best. Flowers scarlet crimson, bloom in profusion for months at a time.

Thurstonii-Pink shaded white.

Parvifolia-Pure white.

Wettsteini - New coral-red.

Ricinifolia-Pale pink.

Metallica-Waxy white

Weltoniensis-Rosy pink.

Marguerite-Light rose.

Verschaffelti-Bright pink.

Alba Perfecta Grandiflora-White.

Hybrida Richardsonii-Pure white.

Rubella-Pink and white.

Semperflorens Alba-Pure white.

Semperflarens Gigantea Rosea-Clear red.

Manicata-Light pink.

\section{REX BEGONIAS.}

Robert George-Large lobed leaves, rich glossy silver with center bronze green. Edge of leaf is metallic green spotted silver.

Queen Victorin-Bright silver-green of crepe like texture, reddish veins and fluted edge.

Desfontaines-Heavy leaves with palmate'center dotted with silver, edge of olive green with bright silver dotting.

Madam Treyve-Upright growing true Rex. Narrow edge of brownish plum color about a very broad soft green zone.

Clementina - Upper surface of leaf beautiful bronze green, with large silver spots. Under side of leaf is bronzy red which makes a fine effect.

Perle Humfeldt-Sharply pointed leaf shows a magnificent combination of silver and bright silky-green and deep maroon.

Louise Erdody-Center dark silver edged with crimson. Leaf has a spiral wind which is very odd.

Beauty of Richmond-Dwarf and compact grower. Leaf bright silver with pretty green about the veins and edge. A splendid Rex.

Mrs. Shepherd-Handsome silvery white leaf with a border of mottled green, making a most showy effect.

For gardens or lawns, you cannot excel the Eufurt Begonia which is used extensively at the National

Soldiers Home of this city. We will make this splendid bedder in quantity at 50 cents per dozen. 


\section{MISCELLANEOUS PLANTS.}

\section{PRICE PER PLANT.}

\section{Pelarganiums in Assortment, Best Kinds, Io Cents Each.}

Trailing Queen Fuchsia, 10 cents.

Asparagus Plumosus, extra large 25 cents.

Tuberoses, 5 cents, 50 cents per dozen.

Gladioli, 5 cents, 50 cents per dozen.

Peperomia Maculosa, 10 cents.

Spotted Calla, 10 cents.

Saxafraga Sarmentosa, 5 cents.

Dwarf Salvia, 5 cents each, 50 cents per dozen.

White Moonflower, 5 cents each, 50 cents per dozen.

Blue Moonflower, 5 cents each, 50 cents per dozen.

Brazilian Plume Plant, 10 cents.

Strobilanthes Dyerianus, 10 cents.

Pylogine Sauvis, 5 cents.

Hardy Primroses-White and yellow, 10 cents.

Anthericum Vitatum Var, 10 cents.

Mexican Primrose, 5 cents.

Lemon Verbena, 5 cents.

Clerodendron Balfouri, 5 cents.

Goldfussia Anisophylla, 5 cents.

Mahernia Odorata, 5 cents.

Ivy, 5 cents.

German Ivy, 5 cents.

Manetta Bicolor, 5 cents.

Achillea, the Pearl, 5 cents.

Violet-Marie Louise, 5 cents.

Violet-Swanley White, 5 cents.

Stapelia Serpentina, 5 cents.

Cuphea, 5 cents.

Pilea, 5 cents.

Evergrcen Sweet-scented Honeysuckle, 10 cents.

Golden-leaved Honeysuckle, 10 cents.

Solanum Jasminoides Grandiflora, 5 cents.

Feverfew, 5 cents.

Wisteria, blue, 10 cents.

Geranium Fernifolia Odorata, 10 cents.

Variegated Dew Plant, 5 cents.

Marguerites, white and yellow, 5 cents.

Nierembergia, 5 cents.

Abutilon Eclipse, 5 cents.

Abutilon Infantia Eulalie, silver-leafed, 10 cents.

Double Petunias, blotched, purple, pink and white, 5 cents.
Weeping Lantana, 10 cents.

Dusty Miller, 5 cents, 50 cents per dozen.

Caladiums, 10 cents, extra large 25 cents.

Santolina, 5 cents.

Vinca Variegata-beautiful silver-leafed vine and trailer for hanging baskets, vases and flowerboxes, nice plants, 10 cents; extra size 15 cents.

Variegated Ground Ivy, 5 cents.

Linum Tygrinum, 10 cents.

Lopesia Rosea, 5 cents.

Lopesia Alba, 5 cents.

Acalphyas-Tricolor, 10 cents.

Acalphyas-Marginata, 10 cents.

Orchid Hyacinth, 5 cents.

Passion Flower, 10 cents. A beautiful vine.

Ageratums, 5 cents.

Achyranthus, 5 cents.

Alyssum-Double white, 5 cents.

Farfugium Grande, 15 cents.

Umbrella Plant, 5 cents.

Hypericum Moseranium, 10 cents.

Hardy Phlox, in variety, 5 cents each, 50c. per doz.

Lantanas, in variety, 5 cents each, 50 cents per doz.

Coleus, best bedding varieties, $3 \mathrm{c}$. each, $30 \mathrm{c}$. per doz.

New Mexican Peperomia, 5 cents.

Libonia Florabunda, 5 cents.

Eranthemum, Pulchellum, 5 cents.

Hardy Plumbago, 10 cents.

Alternantheras, 5 cents.

Hydrangia Otaksa, 10 cents.

Hydrangia Hortensis, 10 cents.

Hydrangia Thomas Hogg, 10 cents.

Tradescantia Multicolor, 5 cents.

Tradescantia Zebrina, 5 cents.

Mesembryanthemum Grandiflora, 5 cents.

Coreopsis Lanceolata Grandiflora, 10 cents.

New Tri-color Cuphea, 5 cents.

Oxalis Rosea, 5 cents.

Cinnamon Vine, 5 cents.

Periwinkle, pink and white, 5 cents.

When ordered by express, larger plants will be sent than can be forwarded through the mails. All plants, either by mail or express, strictly first-elass.

You will readily see that if we sent out a fine illustrated catalogue, which is very expensive in itself, also taking two or three cents postage, our patrons would have to pay for same. We therefore send out this net list, giving our customers the benefit of what we save thereby. both in low price and extra fine quality of stock. 


\section{FINE FERNS.}

No collection of plants is complete without Ferns. The selection we offer embraces the best varieties of this beautiful class of plants, whose diversity and gracefulness of foliage make them peculiarly fitted to combine with other fine plants and flowers.

The popularity of ferns has increased wonderfully in the past few years, and they are now indispensable in all choice floral decorations, and are especially valued for ferneries for room and table decoration.

Where there is moisture and shade most of the varieties succeed well when planted out-of-doors; they delight in a light soil mixed with sand and peat or leaf mould; good drainage must be provided, as stagnant water is fatal to success. Price 10 cents each.

Sword Fern,

Pteris Plumosa,
Pteris Serrulata,

Pteris Cretica,

AND OTHER LEADING VARIETIES.
Pteris Tremula,

Pteris hastata adianthoides.

$$
* *
$$

We have a limited stock of fine, large HARDY ROSES, 3 years old, budded stock, which are so large they can be sent by express only. These are the only budded roses we have. All other roses in this catalogue are on their own roots. Price 25 cents each as follows:

Paul Neyron.

General Jack.

Mme. Chas. Wood.

Mrs. John Laing.

Ulrich Brunner.

Magna Charta.

Also Gloiro De Dijon at 50 cents each.

***

EXTRA LARGE SIZE HARDY PINK, HER MAJESTY, 10 CENTS EACH.

* * *

REMEMBER OUR EXTRA LARGE THREE YEAR OLD BRIDE, BRIDESMAID AND METEOR ROSES AT 25 CENTS EACH.

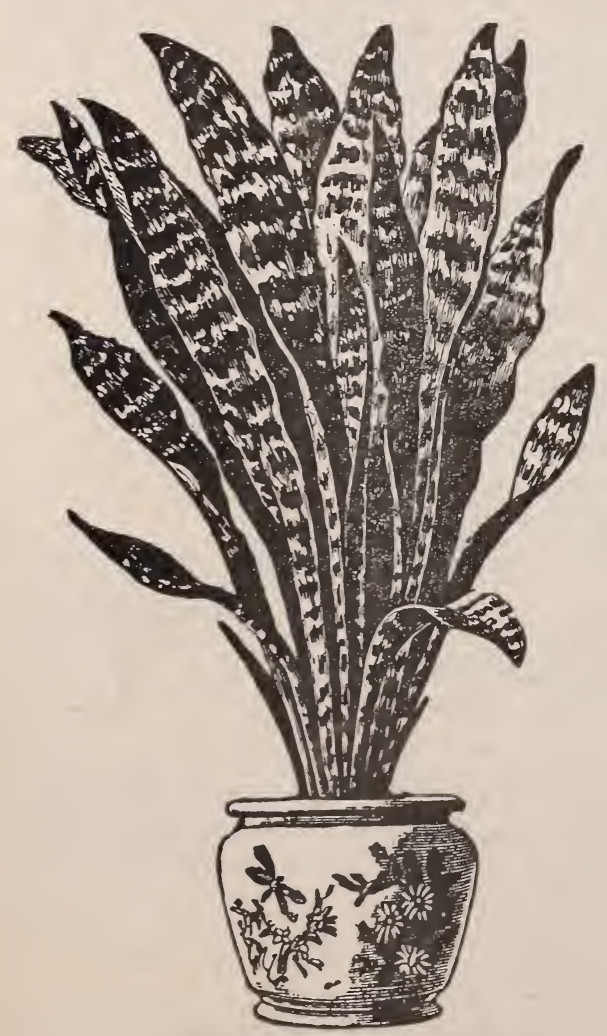

SANSEVERIA ZEALANICA.

$* * 2$

\section{Sanseveria Zealanica.}

\section{A NEW DECORATIVE PLANT OF GREAT BEAUTY} AND VALUE.

A beautiful plant, splendidly adapted for the decoration of drawingorooms and halls, as it stands drouth and dust with impunity. A plant which should be abundantly grown for positions out of the reach of sunlight, where other plants will not thrive. When you consider that it can be placed in any position in any room, and do well, its great usefulness is at once apparent. It has a singular beauty for decorative purposes, which other plants do not possess, and is useful both winter and summer. It is difficult to find a more unique or ornamental plant than this. Good size plants, 10 cents, extra large, 15 cents.

\section{$* *$}

We would recommend to our customers in one vicinity to club together and order by express. Have the goods for all shipped in one box, and on receipt distribute to each person ordering. This will save a great amount in express charges, making the cost nominal to each one.

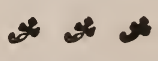

Kindly inform your friends of our generous offer this year, and send in their names, on receipt of which we will be pleased to mail them a copy of this list. 